Article

\title{
Neutrino Emissivity in the Quark-Hadron Mixed Phase
}

\author{
William M. Spinella ${ }^{1}$, Fridolin Weber ${ }^{2,3, *}$ (1) , Milva G. Orsaria ${ }^{4,5}$ (i) and Gustavo A. Contrera $4,5,6$ \\ 1 Department of Sciences, Wentworth Institute of Technology, 550 Huntington Avenue, Boston, MA 02115, \\ USA; spinellaw@wit.edu \\ 2 Department of Physics, San Diego State University, San Diego, CA 92182, USA \\ 3 Center for Astrophysics and Space Sciences, University of California at San Diego, La Jolla, CA 92093, USA \\ 4 National Scientific and Technical Research Council (CONICET), Godoy Cruz 2290, Buenos Aires 1425, \\ Argentina; morsaria@fcaglp.unlp.edu.ar (M.G.O.); contrera@fisica.unlp.edu.ar (G.A.C.) \\ 5 Grupo de Gravitación, Astrofísica y Cosmología, Facultad de Ciencias Astronómicas y Geofísicas, \\ Universidad Nacional de La Plata, La Plata 1900, Argentina \\ 6 Instituto de Física La Plata, CONICET, Universidad Nacional de La Plata, La Plata 1900, Argentina \\ * Correspondence: fweber@sdsu.edu or fweber@ucsd.edu
}

Received: 28 January 2018; Accepted: 4 May 2018; Published: 16 May 2018

\begin{abstract}
In this work we investigate the effect a crystalline quark-hadron mixed phase can have on the neutrino emissivity from the cores of neutron stars. To this end we use relativistic mean-field equations of state to model hadronic matter and a nonlocal extension of the three-flavor Nambu-Jona-Lasinio model for quark matter. Next we determine the extent of the quark-hadron mixed phase and its crystalline structure using the Glendenning construction, allowing for the formation of spherical blob, rod, and slab rare phase geometries. Finally, we calculate the neutrino emissivity due to electron-lattice interactions utilizing the formalism developed for the analogous process in neutron star crusts. We find that the contribution to the neutrino emissivity due to the presence of a crystalline quark-hadron mixed phase is substantial compared to other mechanisms at fairly low temperatures $\left(\lesssim 10^{9} \mathrm{~K}\right)$ and quark fractions $(\lesssim 30 \%)$, and that contributions due to lattice vibrations are insignificant compared to static-lattice contributions. There are a number of open issues that need to be addressed in a future study on the neutrino emission rates caused by electron-quark blob bremsstrahlung. Chiefly among them are the role of collective oscillations of matter, electron band structures, and of gaps at the boundaries of the Brillouin zones on bremsstrahlung, as discussed in the summary section of this paper. We hope this paper will stimulate studies addressing these issues.
\end{abstract}

Keywords: quark matter; hadronic matter; quark deconfinement; neutron star matter; nuclear equation of state; phase transition; crystalline structure; neutrino emissivities

\section{Introduction}

It was shown by Glendenning [1,2] that if electric charge neutrality in a neutron star [3-5] is treated globally rather than locally, the possible first order phase transition from hadronic matter to quark matter in the neutron star core will result in a mixed phase in which both phases of matter coexist. To minimize the total isospin asymmetry energy the two phases will segregate themselves, which results in positively charged regions of hadronic matter and negatively charged regions of quark matter, with the rare phase occupying sites on a Coulomb lattice. The situation is schematically illustrated in Figure 1. Further, the competition between the Coulomb and surface energy densities will cause the matter to arrange itself into energy minimizing geometric configurations $[1,2]$. 

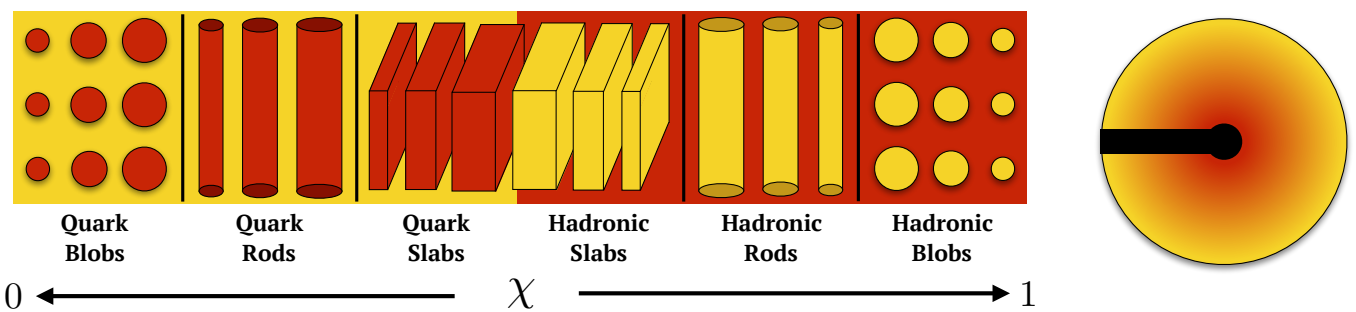

Figure 1. Schematic illustrating the rare phase structures that may form in the quark-hadron mixed phase $[6,7])$. An increase in the volume fraction of quark matter, described by $\chi$, is accompanied by an increase in baryon number density and depth within a neutron star.

The presence of the Coulomb lattice and the nature of the geometric configurations of matter in the quark-hadron mixed phase may have a significant effect on the neutrino emissivity from the core. More specifically, neutrino-antineutrino pairs will be created by the scattering of electrons from these charged lattice structures,

$$
\mathrm{e}^{-}+(Z, A) \rightarrow \mathrm{e}^{-}+(Z, A)+v+\bar{v},
$$

and this will increase the emissivity in the mixed phase. This process is analogous to neutrino-pair bremsstrahlung of electrons in the neutron star crust, where ions exist on a lattice immersed in an electron gas, and for which there exists a large body of work (see, for example [8-14]). The situation is more complicated in the quark-hadron mixed phase, but the operative interaction is still the Coulomb interaction. Thus, to estimate the neutrino-pair Bremsstrahlung of electrons from rare phase structures in the quark-hadron mixed phase we rely heavily on this body of work (particularly [8]). We will refer to this additional mechanism as mixed phase Bremsstrahlung (MPB).

Neutrino emissivity due to the interaction of electrons with a crystalline quark-hadron mixed phase has been previously studied in this manner in [6,15]. In the present work we use a set of nuclear equations of state which are in better agreement with the latest nuclear matter constraints at saturation density than those utilized in [6], and are consistent with the $2.01 \mathrm{M}_{\odot}$ mass constraint set by PSR J0348 + 0432 [16]. To describe quark matter we use the nonlocal SU(3) Nambu-Jona-Lasinio (n3NJL) model discussed in [6,17-21]. The n3NJL parametrization used is given as "Set I" in [22], and is in better agreement with the empirical quark masses than the parametrization utilized in [6]. We consider three geometries for the range of possible structures in the mixed phase including spherical blobs, rods, and slabs, and calculate the associated static lattice contributions to the neutrino emissivity. Phonon contributions to the emissivity for rod and slab geometries are not considered, though a comparison of the phonon and static lattice contributions for spherical blobs is given and indicates that phonon contributions may not be significant. Finally, the extent of the conversion to quark matter in the core was determined in [7], and this allows for a comparison between emissivity contributions from standard and enhanced neutrino emission mechanisms including the direct Urca (DU), modified Urca (MU), and baryon-baryon and quark-quark Bremsstrahlung (NPB) processes, and contributions from electron-lattice interactions. For a detailed summary including the equations and coefficients used for the calculation of the standard and enhanced neutrino emission mechanisms, see [7].

The results for different parametrizations are numerous and qualitatively similar, so the DD2 parametrization will be presented exclusively in this paper. The results of the other parametrizations can be found in [7].

\section{Improved Set of Models for the Nuclear Equation of State}

Hadronic matter is modeled in the framework of the relativistic nonlinear mean-field (RMF) approach [23,24], which describes baryons interacting through the exchange of scalar, vector, and isovector mesons (for details, see $[6,7,25]$ ). The RMF approach is parametrized to reproduce the following properties of symmetric nuclear matter at saturation density $n_{0}$ (see Table 1): the binding 
energy per nucleon $\left(E_{0}\right)$, the nuclear incompressibility $\left(K_{0}\right)$, the isospin asymmetry energy $(J)$, and the effective mass $\left(\mathrm{m}^{*} / \mathrm{m}_{N}\right)$. In addition, the RMF parametrizations used in this work employ a density-dependent isovector-meson-baryon coupling constant that can be fit to the slope of the asymmetry energy $\left(L_{0}\right)$ at $n_{0}$. The scalar- and vector-meson-baryon coupling constants of the density-dependent relativistic mean-field models DD2 and ME2 are fit to properties of finite nuclei $[7,26,27]$. These models are an extension of the standard RMF approach that account for medium effects by making the meson-baryon coupling constants dependent on the local baryon number density [28]. The density-dependence of the meson-baryon coupling constants is given by

$$
g_{i B}(n)=g_{i B}\left(n_{0}\right) f_{i}(x)
$$

where $i \in\{\sigma, \omega, \rho\}, x=n / n_{0}$, and $f_{i}(x)$ provides the functional form for the density dependence. The most commonly utilized ansatz for $f_{i}(x)$ are given by [29]

$$
f_{i}(x)=a_{i} \frac{1+b_{i}\left(x+d_{i}\right)^{2}}{1+c_{i}\left(x+d_{i}\right)^{2}}
$$

for $i \in\{\sigma, \omega\}$, and

$$
f_{\rho}(x)=\exp \left[-a_{\rho}(x-1)\right] .
$$

The nine parameters of the density dependence $\left(a_{\sigma}, b_{\sigma}, c_{\sigma}, d_{\sigma}, a_{\omega}, b_{\omega}, c_{\omega}, d_{\omega}, a_{\rho}\right)$, the values of the meson-nucleon couplings at $n_{0}\left(g_{\sigma N}\left(n_{0}\right), g_{\omega N}\left(n_{0}\right), g_{\rho B}\left(n_{0}\right)\right)$, and the mass of the scalar meson $\left(m_{\sigma}\right)$ are all fit to properties of symmetric nuclear matter at $n_{0}$ and to the properties of finite nuclei including but not limited to binding energies, charge and diffraction radii, spin-orbit splittings, and neutron skin thickness (see $[27,30]$ ).

In addition to the nucleons, hyperons and delta isobars $(\Delta s)$ are also considered in the composition of hadronic matter. The scalar-meson-hyperon coupling constants are fit to the following hypernuclear potentials at saturation (see [7] and references therein),

$$
U_{\Lambda}^{(N)}=-28 \mathrm{MeV}, U_{\Sigma}^{(N)}=+30 \mathrm{MeV}, U_{\Xi}^{(N)}=-18 \mathrm{MeV} .
$$

The vector-meson-hyperon coupling constants are taken to be those given by the ESC08 model in SU(3) symmetry [7,31,32],

$$
g_{\omega \Lambda}=g_{\omega \Sigma} \approx 0.79 g_{\omega N}, g_{\omega \Xi} \approx 0.59 g_{\omega N} .
$$

The scalar- and vector-meson- $\Delta$ coupling constants are given as follows,

$$
x_{\sigma \Delta}=x_{\omega \Delta}=1.1, x_{\rho \Delta}=1.0 .
$$

Finally, the isovector-meson-hyperon and isovector-meson- $\Delta$ coupling constants are taken to be universal, with the differences in the baryon isospin accounted for by the isospin operator in the lagrangian. 
Table 1. Properties of symmetric nuclear matter at saturation density for the hadronic parametrizations of this work.

\begin{tabular}{ccccc}
\hline Saturation Property & SWL [7] & GM1L [1,7] & DD2 [26] & ME2 [27] \\
\hline$n_{0}\left(\mathrm{fm}^{-3}\right)$ & 0.150 & 0.153 & 0.149 & 0.152 \\
$E_{0}(\mathrm{MeV})$ & -16.00 & -16.30 & -16.02 & -16.14 \\
$K_{0}(\mathrm{MeV})$ & 260.0 & 300.0 & 242.7 & 250.9 \\
$m^{*} / m_{N}$ & 0.70 & 0.70 & 0.56 & 0.57 \\
$J(\mathrm{MeV})$ & 31.0 & 32.5 & 32.8 & 32.3 \\
$L_{0}(\mathrm{MeV})$ & 55.0 & 55.0 & 55.3 & 51.3 \\
\hline
\end{tabular}

\section{Crystalline Structure of the Quark-Hadron Mixed Phase}

A mixed phase of hadronic and quark matter will arrange itself so as to minimize the total energy of the phase. Under the condition of global charge neutrality, this is the same as minimizing the contributions to the total energy due to phase segregation, which includes the surface and Coulomb energy contributions. Expressions for the Coulomb $\left(\epsilon_{C}\right)$ and surface $\left(\epsilon_{S}\right)$ energy densities can be written as $[1,2]$

$$
\begin{aligned}
\mathcal{E}_{C} & =2 \pi e^{2}\left[q_{H}(\chi)-q_{Q}(\chi)\right]^{2} r^{2} x f_{D}(x), \\
\mathcal{E}_{S} & =D x \alpha(\chi) / r,
\end{aligned}
$$

where $q_{H}\left(q_{Q}\right)$ is the hadronic (quark) phase charge density, $r$ is the radius of the rare phase structure, and $\alpha(\chi)$ is the surface tension between the two phases. The parameter $\chi$, which varies between 0 and 1 , represents the volume fraction of quark matter at a given density. The quantities $x$ and $f_{D}(x)$ in (8) are defined as

$$
x=\min (\chi, 1-\chi)
$$

and

$$
f_{D}(x)=\frac{1}{D+2}\left[\frac{1}{D-2}\left(2-D x^{1-2 / D}\right)+x\right],
$$

where $D$ is the dimensionality of the lattice. The phase rearrangement process will result in the formation of geometrical structures of the rare phase distributed in a crystalline lattice that is immersed in the dominant phase (see Figure 1). The rare phase structures are approximated for convenience as spherical blobs, rods, and slabs [1,2]. The spherical blobs occupy sites in a three dimensional $(D=3)$ body centered cubic $(\mathrm{BCC})$ lattice, the rods in a two dimensional $(D=2)$ triangular lattice, and the slabs in a simple one dimensional $(D=1)$ lattice [8]. At $\chi=0.5$ both hadronic and quark matter exist as slabs in the same proportion, and at $\chi>0.5$ the hadronic phase becomes the rare phase with its geometry evolving in reverse order (from slabs to rods to blobs).

Direct determination of the surface tension of the quark-hadron interface is problematic because of difficulties in constructing a single theory that can accurately describe both hadronic matter and quark matter. Therefore, we employ an approximation proposed by Gibbs where the surface tension is taken to be proportional to the difference in the energy densities of the interacting phases [1,2],

$$
\alpha(\chi)=\eta L\left[\mathcal{E}_{Q}(\chi)-\mathcal{E}_{H}(\chi)\right],
$$

where $L$ is proportional to the surface thickness which should be on the order of the range of the strong interaction $(1 \mathrm{fm})$, and $\eta$ is a proportionality constant. In this work we maintain the energy density proportionality but set the parameter $\eta=0.08$ so that the surface tension falls below $70 \mathrm{MeV} \mathrm{fm}^{-2}$ for all parametrizations, a reasonable upper limit for the existence of a quark-hadron mixed phase [33]. The surface tension as a function of $\chi$ is given in Figure 2 for the nuclear DD2 parametrization, introduced in Section 2. 


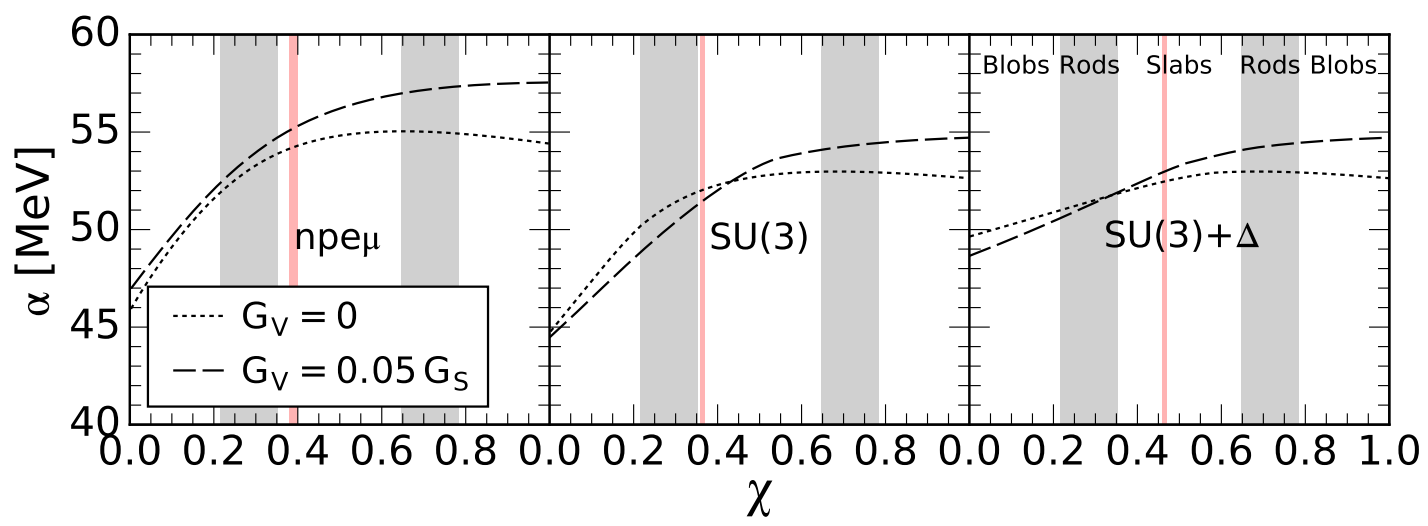

Figure 2. Surface tension $\alpha$ in the quark-hadron mixed phase for the DD2 parametrization [7]. The red shading indicates the range for the maximum quark fraction $\chi_{\max }$ for the two values of the quark vector coupling constant $G_{V}$. (Left panel) Only nucleons and leptons are included in the hadronic phase. (Center panel) Hyperons are included in the hadronic phase. (Right panel) Delta isobars are included in addition to hyperons in the hadronic phase. Similar figures for the SWL, GM1L, and ME2 parametrizations can be found in Reference [7].

We note that, in this work, we restricted ourselves to considering $G_{V}$ values that are in the range of $0<G_{V}<0.05 G_{S}$, as this choice leads to gravitational masses of neutron stars with quark-hybrid compositions that satisfy the $2 M_{\odot}$ constraint. Exploring the possibility of larger $G_{V}$ values would certainly be worthwhile, but this is beyond the scope of this work.

The size of the rare phase structures is given by the radius $(r)$ and is determined by minimizing the sum of the Coulomb and surface energies, $\partial\left(\mathcal{E}_{C}+\mathcal{E}_{S}\right) / \partial r$, and solving for $r[1,2]$,

$$
r(\chi)=\left(\frac{D \alpha(\chi)}{4 \pi e^{2} f_{D}(\chi)\left[q_{H}(\chi)-q_{Q}(\chi)\right]^{2}}\right)^{\frac{1}{3}} .
$$

Rare phase structures are centered in the primitive cell of the lattice, taken to be a Wigner-Seitz cell of the same geometry as the rare phase structure. The Wigner-Seitz cell radius $R$ is set so that the primitive cell is charge neutral,

$$
R(\chi)=r x^{-1 / D} .
$$

Figure 3 shows $r$ and $R$ as a function of the quark fraction in the mixed phase. Both $r$ and $R$ increase with an increase in the baryonic degrees of freedom, particularly when $\chi \lesssim 0.5$ and the vector interaction is included. Note that the blob radius should vanish for $\chi \in\{0,1\}$, but does not due to the approximate nature of the geometry function $f_{D}(\chi)$ [15]. The number density of rare phase blobs will be important for calculating the phonon contribution to the emissivity. Since there is one rare phase blob per Wigner-Seitz cell, the number density of rare phase blobs $\left(n_{b}\right)$ is simply the reciprocal of the Wigner-Seitz cell volume,

$$
n_{b}=\left(4 \pi R^{3} / 3\right)^{-1} \text {. }
$$

The density of electrons in the mixed phase is taken to be uniform throughout. Charge densities in both the rare and dominant phases are also taken to be uniform, an approximation supported by a recent study by Yasutake et al. [33]. The uniformity of charge in the rare phase also justifies the use of the nuclear form factor $(F(q))$ presented in Section 4 . The total charge number per unit volume $\left(|Z| / V_{\text {Rare }}\right)$ of the rare phase structures is given in Figure 4. 


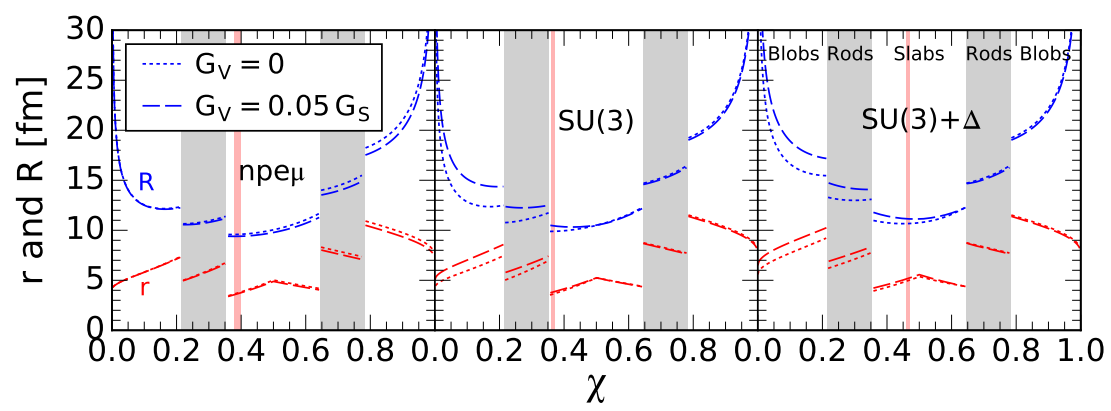

Figure 3. Radius of the rare phase structure $r$ and Wigner-Seitz cell $R$ in the quark-hadron mixed phase for the DD2 parametrization [7]. See Figure 2 for additional details. Similar figures for the SWL, GM1L, and ME2 parametrizations can be found in Reference [7].

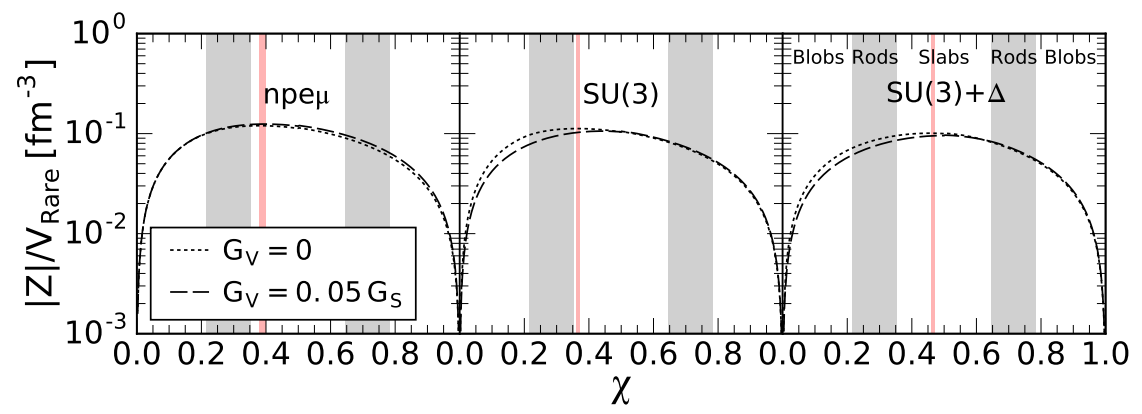

Figure 4. Charge number per unit volume of the rare phase structures for the DD2 parametrization [7]. See Figure 2 for additional details. Similar figures for the SWL, GM1L, and ME2 parametrizations can be found in Reference [7].

\section{Neutrino Emissivity Due to a Crystalline Quark-Hadron Lattice}

We begin this section with a brief discussion of the neutrino emissivity due to a crystalline quark-hadron lattice [6]. Modeling the complex interactions of electrons with a background of neutrons, protons, hyperons, muons, and quarks is an exceptionally complicated problem. However, to make a determination of the neutrino emissivity that is due to electron-lattice interactions in the quark-hadron mixed phase we need only consider the Coulomb interaction between them. This simplifies the problem greatly, as a significant body of work exists for the analogous process of electron-ion scattering that takes place in the crusts of neutron stars.

To determine the state of the lattice in the quark-hadron mixed phase we use the dimensionless ion coupling parameter given by

$$
\Gamma=\frac{Z^{2} e^{2}}{R k_{B} T}
$$

Below $\Gamma_{\text {melt }}=175$ the lattice behaves as a Coulomb liquid, and above as a Coulomb crystal [34,35]. It was shown in Reference [15] that the emissivity due to electron-blob interactions in the mixed phase was insignificant compared to other contributions at temperatures above $T \gtrsim 10^{10} \mathrm{~K}$. Therefore, in this work we consider temperatures in the range $10^{7} \mathrm{~K} \leq T \leq 10^{10} \mathrm{~K}$. At these temperatures the value of the ion coupling parameter is well above $\Gamma_{\text {melt }}$, and so the lattice in the quark-hadron mixed phase is taken to be a Coulomb crystal. 
To account for the fact that the elasticity of scattering events is temperature dependent we need to compute the Debye-Waller factor, which is known for spherical blobs only and requires the plasma frequency and temperature given by

$$
\begin{gathered}
\omega_{p}=\sqrt{\frac{4 \pi Z^{2} e^{2} n_{b}}{m_{b}},} \\
T_{p}=\frac{\hbar \omega_{p}}{k_{B}},
\end{gathered}
$$

where $m_{b}$ is the mass of a spherical blob [8]. The Debye-Waller factor is then given by

$$
W(q)= \begin{cases}\frac{a q^{2}}{8 k_{e}^{2}}\left(1.399 \mathrm{e}^{-9.1 t_{p}}+12.972 t_{p}\right) & \text { spherical blobs } \\ 0 & \text { rods and slabs }\end{cases}
$$

where $q=|\boldsymbol{q}|$ is a phonon or scattering wave vector, $a=4 \hbar^{2} k_{e}^{2} /\left(k_{B} T_{p} m_{b}\right)$, and $t_{p}=T / T_{p}[8,36]$. In order to smooth out the charge distribution over the radial extent of the rare phase structure we adopt the nuclear form factor given in [8],

$$
F(q)=\frac{3}{\left(q R^{3}\right)}[\sin (q R)-q R \cos (q R)] .
$$

Screening of the Coulomb potential by electrons is taken into account by the static dielectric factor $\epsilon(q, 0)=\epsilon(q)$, given in [10]. However, the charge number of the rare phase structures is high and the electron number density is low, so setting this factor to unity has no noticeable effect on the calculated neutrino emissivity. Finally, the effective interaction is given by [8]

$$
V(q)=\frac{4 \pi e \rho_{Z} F(q)}{q^{2} \epsilon(q, 0)} \mathrm{e}^{-W(q)}
$$

General expressions for the neutrino emissivity due to the MPB electron-lattice interactions were derived by Haensel et al. [37] for spherical blobs and by Pethick et al. [14] for rods and slabs,

$$
\begin{aligned}
& \epsilon_{\mathrm{MPB}}^{\text {blobs }} \approx 5.37 \times 10^{20} n T_{9}^{6} \mathrm{Z}^{2} L \mathrm{erg} \mathrm{s}^{-1} \mathrm{~cm}^{-3}, \\
& \epsilon_{\mathrm{MPB}}^{\text {rods,slabs }} \approx 4.81 \times 10^{17} k_{e} T_{9}^{8} J \mathrm{erg} \mathrm{s}^{-1} \mathrm{~cm}^{-3},
\end{aligned}
$$

where $L$ and $J$ are dimensionless quantities that scale the emissivities. Both $L$ and $J$ contain a contribution due to the static lattice (Bragg scattering), but we consider the additional contribution from lattice vibrations (phonons) for spherical blobs, so $L=L_{\mathrm{sl}}+L_{\mathrm{ph}}$. We note that the $T^{8}$ temperature dependence in Equation (23) is somewhat deceiving since the $J$ factor also depends on temperature and, for a wide range of parameters, is proportional to $1 / T^{2}$. In effect, the neutrino emissivity $\epsilon_{\mathrm{MPB}}^{\text {rods, slabs }}$ is therefore proportional to $T^{6}$.

\subsection{Phonon Contribution to Neutrino Emissivity}

The expressions for determining the neutrino emissivity due to interactions between electrons and lattice vibrations (phonons) in a Coulomb crystal, with proper treatment of multi-phonon processes, were obtained by Baiko et al. [38] and simplified by Kaminker et al. [8]. The phonon contribution to the emissivity is primarily due to Umklapp processes in which a phonon is created (or absorbed) by an electron that is simultaneously Bragg reflected, resulting in a scattering vector $q$ that lies outside the first Brillouin zone, $q_{0} \gtrsim\left(6 \pi^{2} n_{b}\right)^{1 / 3}[39,40]$, where $n_{b}$ is given by Equation (15). 
The contribution to MPB due to phonons is contained in $L_{\mathrm{ph}}$ and given by Equation (21) in [8],

$$
L_{\mathrm{ph}}=\int_{y_{0}}^{1} d y \frac{S_{\mathrm{eff}}(q)|F(q)|^{2}}{y|\epsilon(q, 0)|^{2}}\left(1+\frac{2 y^{2}}{1-y^{2}} \ln y\right),
$$

where $y=q /\left(2 k_{e}\right)$, and the lower integration limit $y_{0}$ excludes momentum transfers inside the first Brillouin zone. The structure factor $S_{\text {eff }}$ is given by (24) and (25) in [8]),

$$
\begin{gathered}
S_{\text {eff }}(q)=189\left(\frac{2}{\pi}\right)^{5} \mathrm{e}^{-2 W} \int_{0}^{\infty} d \xi \frac{1-40 \xi^{2}+80 \xi^{4}}{\left(1+4 \xi^{2}\right)^{5} \cosh ^{2}(\pi \xi)} \times\left(\mathrm{e}^{\Phi(\xi)}-1\right), \\
\Phi(\xi)=\frac{\hbar q^{2}}{2 m_{b}}\left\langle\frac{\cos \left(\omega_{s} t\right)}{\omega_{s} \sinh \left(\hbar \omega_{s} / 2 k_{B} T\right)}\right\rangle,
\end{gathered}
$$

where $\xi=t k_{B} T / \hbar$ and $\langle\ldots\rangle$ denotes averaging over phonon frequencies and modes,

$$
\left\langle f_{S}(\boldsymbol{k})\right\rangle=\frac{1}{3 V_{B}} \sum_{S} \int_{V_{B}} d \boldsymbol{k} f_{S}(\boldsymbol{k}) .
$$

It is assumed that there are three phonon modes $s$, two linear transverse and one longitudinal. The frequencies of the transverse modes are given by $\omega_{t_{i}}=a_{i} k$, where $i=1,2, a_{1}=0.58273$, and $a_{2}=0.32296$. The frequency of the longitudinal mode $\omega_{l}$ is determined by Kohn's sum rule, $\omega_{l}^{2}=\omega_{p}^{2}-\omega_{t_{1}}^{2}-\omega_{t_{2}}^{2}[41]$.

Umklapp processes proceed as long as the temperature $T_{\text {Umklapp }} \gtrsim T_{p} Z^{1 / 3} e^{2} /(\hbar c)$, below which electrons can no longer be treated in the free electron approximation [39]. This limits the phonon contribution to the neutrino emissivity to only a very small range in temperature for a crystalline quark-hadron mixed phase (see Figure 5), and renders it negligible compared to the static lattice contribution as will be shown in the next section.

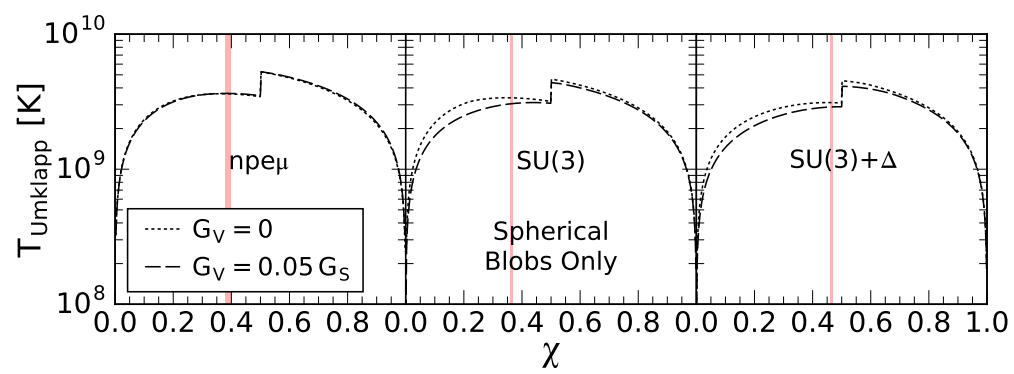

Figure 5. Temperature below which Umklapp processes are frozen out ( $\left.T_{\text {Umklapp }}\right)$, and contributions to the neutrino emissivity due to electron-phonon interactions become negligible for the DD2 parametrization [7]. See Figure 2 for additional details. Similar figures for the SWL, GM1L, and ME2 parametrizations can be found in [7].

\subsection{Static Lattice Contribution to Neutrino Emissivity}

Pethick and Thorsson [14] found that with proper handling of electron band-structure effects the static lattice contribution to the neutrino emissivity in a Coulomb crystal was significantly reduced compared to calculations performed in the free electron approximation. Kaminker et al. [8] presented simplified expressions for calculating the static lattice contribution $\left(L_{\mathrm{sl}}\right)$ using the formalism developed in [14]. The dimensionless quantities $L_{\mathrm{sl}}$ and $J$ that scale the neutrino emissivities for spherical blobs and rods/slabs, respectively, are given by

$$
L_{\mathrm{sl}}=\frac{1}{12 Z} \sum_{K \neq 0} \frac{\left(1-y_{K}^{2}\right)}{y_{K}^{2}} \frac{|F(K)|^{2}}{|\epsilon(K)|^{2}} I\left(y_{K}, t_{V}\right) \mathrm{e}^{-2 W(K)}
$$


and

$$
J=\sum_{K \neq 0} \frac{y_{K}^{2}}{t_{V}^{2}} I\left(y_{K}, t_{V}\right),
$$

where $K=|K|$ is a scattering vector and restricted to linear combinations of reciprocal lattice vectors, $y_{K}=K /\left(2 k_{e}\right), t_{V}=k_{B} T /\left[|V(K)|\left(1-y_{K}^{2}\right)\right]$, and $I\left(y_{K}, t_{V}\right)$ is given by Equation (39) in [8]. The sum over $K$ in (28) and (29) terminates when $K>2 k_{e}$, prohibiting scattering vectors that lie outside the electron Fermi surface.

\section{Neutrino Emissivity Results}

The neutrino emissivities due to MPB and the additional emissivity mechanisms are given in Figures 6 and 7 for $G_{V}=0$ and $G_{V}=0.05 G_{S}$ respectively at temperatures between $10^{7} \mathrm{~K}$ and $10^{10} \mathrm{~K}$. The MPB emissivity is for most of the mixed phase the weakest of the emissivity mechanisms, peaking at low $\chi$ (at $\chi \lesssim 0.05$ the MPB emissivity may be overestimated due to the limitations of the dimensionality function), and appears to be slightly larger when hyperons and $\Delta \mathrm{s}$ are included in the composition. Including the vector interaction $\left(G_{V}=0.05 G_{S}\right)$ also results in a slight increase in the MPB emissivity. Both additional baryonic degrees of freedom and inclusion of the vector interaction delay the onset of the quark-hadron phase transition, and therefore it may be concluded that the greater the density in the mixed phase, the greater the contribution to the emissivity from MPB. The MPB emissivity is most comparable to the modified Urca emissivity, particularly at $10^{8}-10^{9} \mathrm{~K}$.

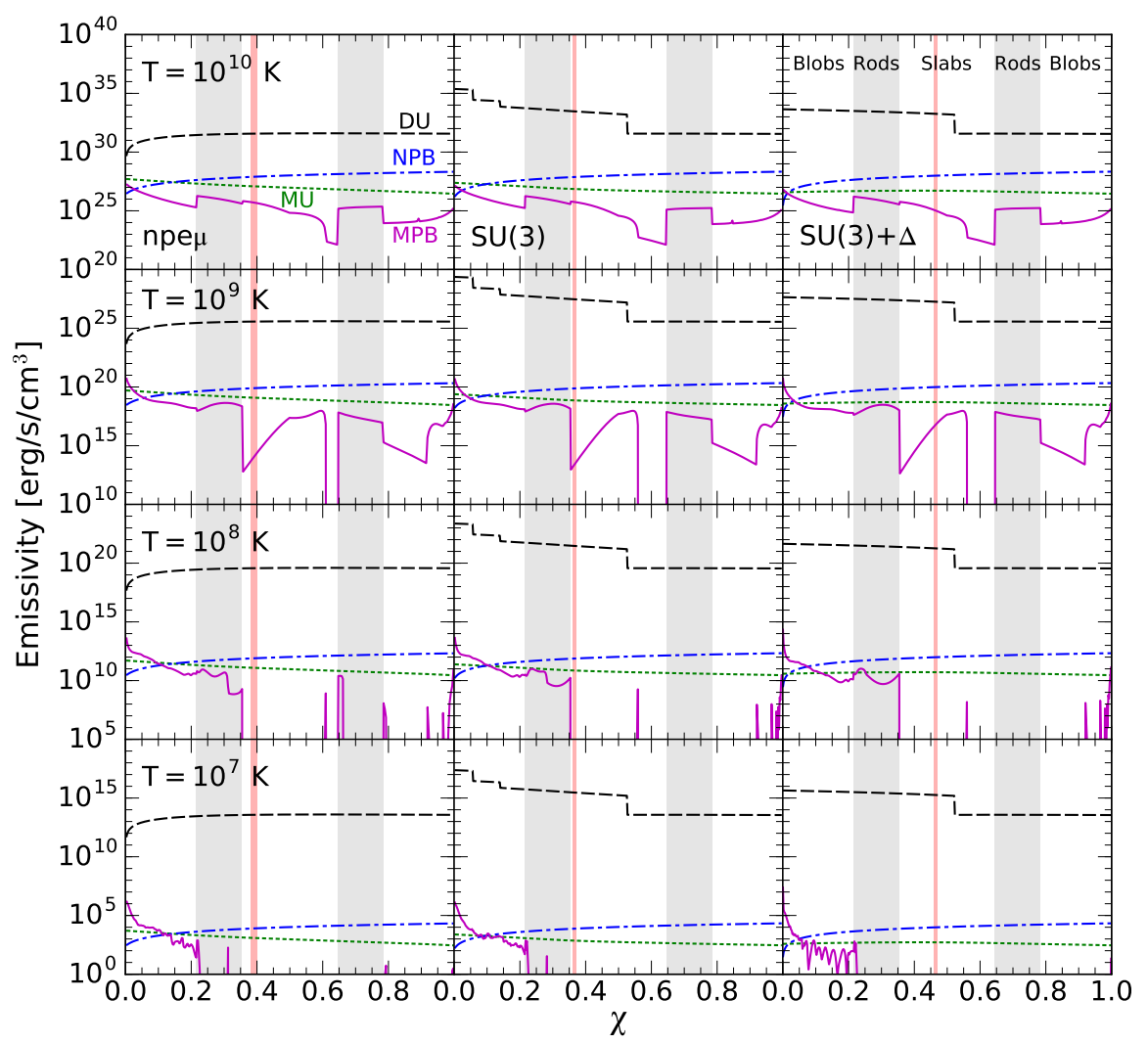

Figure 6. Neutrino emissivity in the quark-hadron mixed phase for the DD2 parametrization with $G_{V}=0$ [7]. Contributions due to mixed phase Bremsstrahlung (MPB), nucleon-nucleon and quark-quark neutrino pair Bremsstrahlung (NPB), the nucleon and quark modified Urca processes (MU), and the hyperon and quark direct Urca (DU) processes are included. See Figure 2 for additional details. Similar figures for the SWL, GM1L, and ME2 parametrizations can be found in [7]. 


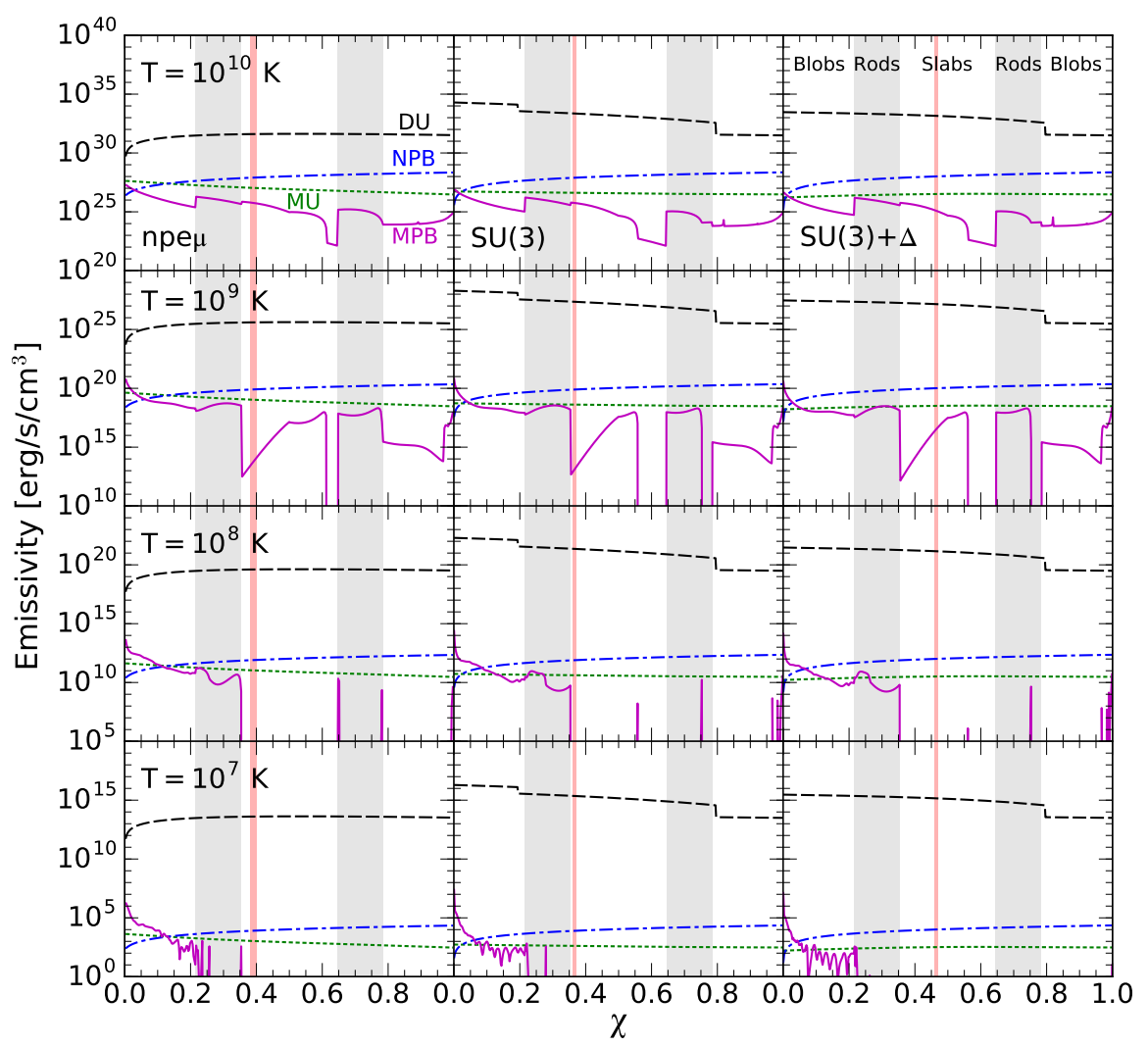

Figure 7. Neutrino emissivity in the quark-hadron mixed phase for the DD2 parametrization with $G_{V}=0.05 G_{S}$ [7]. Contributions due to mixed phase Bremsstrahlung (MPB), nucleon-nucleon and quark-quark neutrino pair Bremsstrahlung (NPB), the nucleon and quark modified Urca processes (MU), and the hyperon and quark direct Urca (DU) processes are included. See Figure 2 for additional details. Similar figures for the SWL, GM1L, and ME2 parametrizations can be found in [7].

Electron-phonon interactions contribute to the MPB emissivity when the mixed phase consists of spherical blobs $(\chi \lesssim 0.21$ and $\chi \gtrsim 0.79)$ and only when $T>T_{\text {Umklapp }}$ (Figure 5), which for the given choices of temperature implies $T=10^{10} \mathrm{~K}$. Figure 8 shows that the static-lattice contribution to the MPB emissivity dominates the phonon contribution rendering it negligible, particularly at quark fractions relevant to the neutron stars of this work $(\chi<0.5)$. Therefore, the MPB emissivity is almost entirely due to the static-lattice contribution (Bragg scattering).

Equations (28) and (29) indicate that the static-lattice contribution to the MPB emissivity is calculated as a sum over scattering vectors $K$ that satisfy $K<2 k_{e}$. At the onset of the mixed phase $k_{e}$ and $N_{K}$ are at a maximum, but as the quark-hadron phase transition proceeds the negatively charged down and strange quarks take over the process of charge neutralization. Thus, the electron number density and consequently $k_{e}$ continue to decrease at about the same rate as before the start of the mixed phase. This leads to the steep decline in $N_{K}$ with increasing $\chi$ for $\chi<0.5$ shown in Figure 9 . Further, the rod and slab dimensionality drastically reduces the number of available scattering vectors which contributes to the decrease of the MPB emissivity in those phases, particularly in the slab phase. However, (29) shows that the MPB emissivity from rod and slab phases is dependent on $T^{8}$, rather than $T^{6}$ for the blob phase, and this explains the dramatic decrease in the MPB emissivity with decreasing temperature.

Direct Urca processes dominate the mixed phase neutrino emissivity at all temperatures, with contributions from the $\Lambda$ hyperon DU process $(\Lambda \rightarrow p e \bar{v})$ operating beyond $\chi_{\max }$. Nucleonic DU processes do not operate for any of the parametrizations considered in this work [7]. The hyperon DU process emissivities can be identified as any contribution with an emissivity above that for the 
quark DU process in the nре $\mu$ composition, and are shown to step down in the mixed phase, vanishing prior to the onset of a pure quark phase. In the absence of the hyperonic DU process, the quark DU process would still dominate the Bremsstrahlung and modified Urca processes unless curtailed by the presence of color superconductivity.

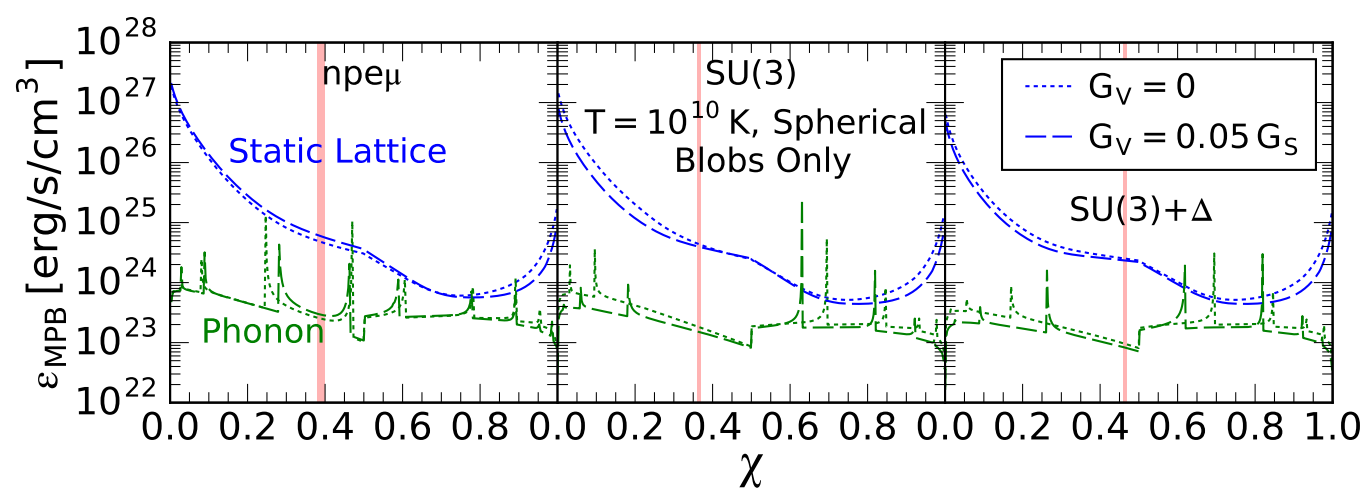

Figure 8. Comparison of the static lattice and phonon contributions to the neutrino emissivity at $T=10^{10} \mathrm{~K}$ for the spherical blob geometry only and the DD2 parametrization [7].

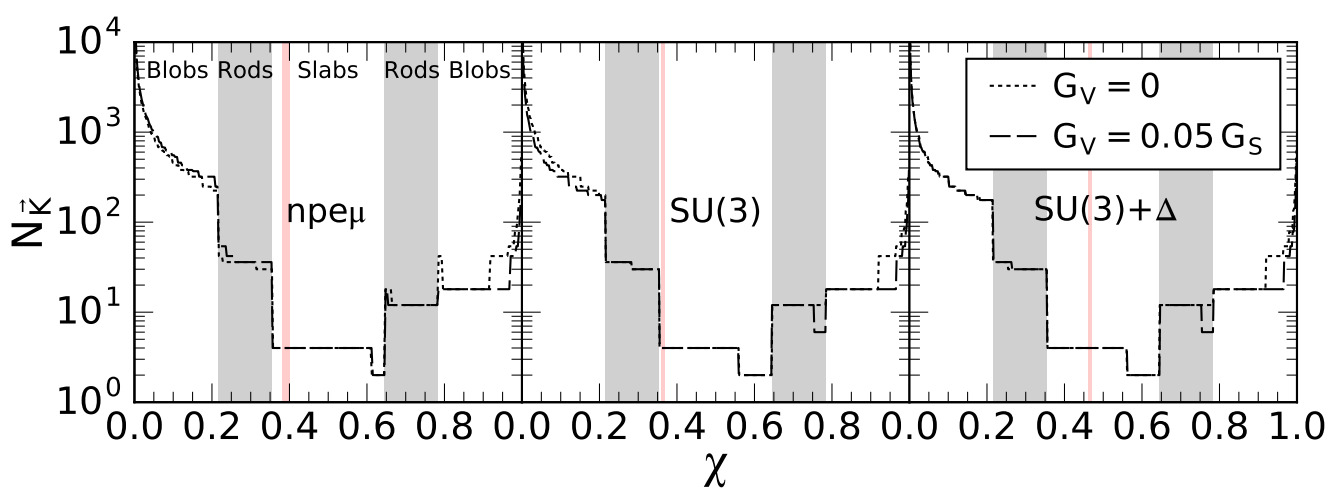

Figure 9. The number of scattering vectors that satisfy the condition $K<2 k_{e}$ as a function of the quark fraction [7] (see Figure 2 for additional details). Similar figures for the SWL, GM1L, and ME2 parametrizations can be found in [7].

\section{Discussion and Summary}

In this work we determined that quark blob, rod, and slab structures may exist in a crystalline quark-hadron mixed phase. The study is based on relativistic mean-field equations of state which are used to model hadronic matter and a nonlocal extension of the three-flavor Nambu-Jona-Lasinio model for quark matter. We determined the neutrino emissivities that may result from the elastic scattering of electrons off these quark structures (mixed phase Bremsstrahlung (MBP)), and compared them to standard neutrino emissivity processes that may operate in the mixed phase as well.

We found that the emissivity from the MPB process is comparable to that of the modified Urca process at low volume fractions of quark matter, $\chi$, and in the temperature range of $10^{8} \mathrm{~K} \lesssim T \lesssim 10^{9} \mathrm{~K}$. The MPB emissivity was found to increase with the inclusion of the vector interaction among quarks and with additional baryonic degrees of freedom in the form of hyperons and $\Delta$ baryons [7], both of which lead to an increase in the quark-hadron phase transition density and a higher density core. Further, contributions to the MPB emissivity from phonons were shown to be negligible compared to those from Bragg scattering. Finally, baryonic and quark DU processes were shown to operate in the mixed phase and dominate all other neutrino emissivity mechanisms. 
Since it is believed that the hypothetical quark-hadron lattice structures in the core regions of neutron stars are qualitatively reminiscent to the hypothesized structures in the crustal regions of neutron stars $[1,2,42-44]$, we have adopted the Bremsstrahlung formalism developed in the literature for the crustal regions of neutron stars to assess the neutrino emission rates resulting from electron-quark blob (rod, slab) scattering in the cores of neutron stars with quark-hybrid compositions. Because of the complexity of the problem, however, there are several issues that need to be studied further in order to develop refined estimates of the neutrino emission rates presented in this paper. The remaining part of this section is devoted to this topic.

Properties of the sub-nuclear crustal region: Thehypothetical structures in the crustal regions of neutron stars range in shape from spheres to rods to slabs at mass densities $10^{14} \mathrm{~g} \mathrm{~cm}^{-3} \lesssim \rho \lesssim$ $1.5 \times 10^{14} \mathrm{~g} \mathrm{~cm}^{-3}$, which is just below the nuclear saturation density of $2.5 \times 10^{14} \mathrm{~g} \mathrm{~cm}^{-3}$. At densities where the nuclei are still spherical in such matter, the chemical potential of the electrons is $\mu_{e} \sim 80 \mathrm{MeV}$ and the atomic number of the nuclei is $Z \sim 50$ [45]. The corresponding Wigner-Seitz cell has a radius of $R \sim 18 \mathrm{fm}$, and the radius of the nucleus inside the cell is $r \sim 9 \mathrm{fm}$ [45]. The electrons moving in the crystalline lattice formed by the ions are highly relativistic and strongly degenerate. The ion coupling parameter, defined in Equation (16), is $\Gamma \sim 2.3 \times 10^{12} / T$, and the melting temperature $T_{\text {melt }} \sim(Z e)^{2} /\left(R k_{B} \Gamma_{\text {melt }}\right)$ has a value of $T_{\text {melt }} \sim 1.3 \times 10^{10} \mathrm{~K}$.

Properties of the quark-hadron lattice: The size of the Wigner-Seitz cells associated with spherical quark blobs in the crystalline quark-hadron phase is similar to the size of the Wigner-Seitz cells in the crust. (Here, we do not consider the crystalline phases made of quark rods and quark slabs since they contribute much less to Bremsstrahlung because of the much smaller number of electrons in those phases.) For spherical quark blobs at the onset of quark deconfinement, which occurs in our models at densities of around three times nuclear saturation, $3 n_{0}$, the electron chemical potential is $\mu_{e}=k_{e} \sim 140 \mathrm{MeV}$. Hence, like at sub-nuclear densities, the electrons are ultra-relativistic $\left(\hbar k_{e} / m c^{2}=275\right)$ and strongly degenerate. The electron degeneracy temperature is around $T_{F} \sim 1.6 \times 10^{12} \mathrm{~K}$, which is much higher than the temperature range $\left(\lesssim 10^{10} \mathrm{~K}\right)$ considered in this paper. From the results shown in Figure 3, one sees that the radii of the Wigner-Seitz cells containing spherical quark-blobs are around $R \sim 12 \mathrm{fm}$ and that the quark blobs inside the cells have radii of $r \sim 8 \mathrm{fm}$. The density of the Wigner-Seitz cells is $\left(4 \pi R^{3} / 3\right)^{-1} \sim 1.4 \times 10^{-4} \mathrm{fm}^{-3}$ and the atomic number of the quark blobs inside the Wigner-Seitz cell is around $Z \sim 200$.

Plasma temperature and melting temperature: The ion (quark blob) coupling parameter $\Gamma=(Z e)^{2} /\left(R k_{B} T\right)$ is given by $\Gamma=6.7 \times 10^{13} / T$ and the melting temperature of the ion crystal is $T_{\text {melt }}=(Z e)^{2} /\left(R k_{B} 172\right) \sim 4 \times 10^{11} \mathrm{~K}$. Here we have used $\Gamma_{\text {melt }}=174$ for which a solid is expected to form $[34,35]$. Since the melting temperature of the quark crystal exceeds $10^{11} \mathrm{~K}$ the quark blobs are expected to be in the crystalline phase at all temperatures $\left(\lesssim 10^{10} \mathrm{~K}\right)$ considered in our study. The plasma temperature of the system follows from $T_{P}=7.83 \times 10^{9} \sqrt{Z Y_{e} \rho_{12} / A_{i}}$, where $Y_{e}=n_{e} / n_{b}$ is the number of electrons per baryon, $n_{e}$ the number density of electrons, $n_{b}$ the number density of baryons, and $\rho_{12}$ the mass density in units of $10^{12} \mathrm{~g} / \mathrm{cm}^{3}$. For quark blobs with mass numbers of $A \sim 2000$, atomic number $Z \sim 200$, and $Y_{e} \sim 0.06$ one obtains a plasma temperature of $T_{P} \sim 2 \times 10^{10} \mathrm{~K}$.

Electron-phonon scattering and Umklapp processes: In an Umklapp process, the electron momentum transfer in a scattering event, $\hbar \vec{q}$, lies outside the first Brillouin zone, that is, $\hbar q \gtrsim \hbar q_{0}$. This is in contrast to the normal processes where $\hbar \vec{q}$ remains in the first Brillouin zone and $\hbar q \lesssim \hbar q_{0}$, where $q_{0} \approx\left(6 \pi^{2} n_{\mathrm{Blob}}\right)^{1 / 3}$. For the quark-blob phase we find $\hbar q_{0} \sim 30 \mathrm{MeV}$ so that $q_{0} /\left(2 k_{e}\right) \sim$ 0.13 for the quark-blob lattice, which is of the same order of magnitude as for the crust where $q_{0} /\left(2 k_{e}\right)=(4 Z)^{-1 / 3} \sim 0.01$ [8]. The temperature below which the Umklapp processes are frozen out is $T_{\text {Umklapp }} \sim T_{P} Z^{1 / 3} e^{2} \sim 8 \times 10^{8} \mathrm{~K}$, with the plasma temperature $T_{P}$ given just above. We find that the temperatures obtained for $T_{\text {Umklapp }}, T_{P}$, and $T_{\text {melt }}$ in the quark-blob phase are rather similar to their counterparts in the nuclear lattice just below nuclear saturation density, namely $T_{\text {Umklapp }} \sim 10^{8}$ $\mathrm{K}, T_{P} \sim 10^{9} \mathrm{~K}$, and $T_{\text {melt }} \sim 10^{10} \mathrm{~K}$. In our study, both the Umklapp process and the normal process are taken into account since temperatures in the range of $10^{6} \mathrm{~K}<T<10^{10} \mathrm{~K}$ are considered. 
Debye-Waller factor: The effective interaction between electrons and quark blobs depends on the thermal quark-blob lattice vibrations which effectively smear-out the quark blob charges. This feature is taken into account via the Debye-Waller factor given in Equation (19). Since estimates for the Debye-Waller factor are only known for spherical blob structures, the Debye-Waller may be the largest source of uncertainty in our study.

Role of electron band structure effects: It has been shown in [46] that gaps in the electron dispersion relation at the boundaries of Brillouin zones can noticeably reduce the static lattice contribution. For point-like quark blobs with atomic number $Z$ and for the smallest reciprocal lattice vector in a bcc lattice, we estimate the electron band splitting from $0.018(Z / 60)^{2 / 3} k_{e}$ [46]. This leads to a splitting of $\sim 6 \mathrm{MeV}$ for the quark-blob phase, which is around $1 \mathrm{MeV}$ or more for the nuclear lattice case [46].

Author Contributions: The authors contributed equally to the theoretical and numerical aspects of the work presented in this paper.

Funding: This research was supported by CONICET and UNLP, grant numbers PIP 0714, G 157 and G 140, and by the National Science Foundation (USA), grant numbers PHY-1411708 and PHY-1714068.

Acknowledgments: M.G.O. and G.A.C. thank Consejo Nacional de Investigaciones Científicas y Técnicas (CONICET) and the Universidad Nacional de La Plata (UNLP) for financial support. F.W. is supported by the National Science Foundation (USA).

Conflicts of Interest: The authors declare no conflict of interest.

\section{References}

1. Glendenning, N.K. First-order phase transitions with more than one conserved charge: Consequences for neutron stars. Phys. Rev. D 1992, 46, 1274-1287. [CrossRef]

2. Glendenning, N.K. Phase transitions and crystalline structures in neutron star cores. Phys. Rep. 2001, 342, 393-447. [CrossRef]

3. Page, D.; Reddy, S. Dense Matter in Compact Stars: Theoretical Developments and Observational Constraints. Ann. Rev. Nucl. Part. Sci. 2006, 56, 327-374. [CrossRef]

4. Becker, W. (Ed.) Neutron Stars and Pulsars; Astrophysics and Space Science Library Series 357; Springer: Berlin, Germany, 2009.

5. Buballa, M.; Dexheimer, V.; Drago, A.; Fraga, E.; Haensel, P.; Mishustin, I.; Pagliara, G.; Schaffner-Bielich, J.; Schramm, S.; Sedrakian, A.; et al. EMMI rapid reaction task force meeting on quark matter in compact stars. J. Phys. G Nucl. Part. Phys. 2014, 41, 123001. [CrossRef]

6. Spinella, W.M.; Weber, F.; Contrera, G.A.; Orsaria, M.G. Neutrino emissivity in the quark-hadron mixed phase of neutron stars. Eur. Phys. A 2016, 52, 1-12. [CrossRef]

7. Spinella, W.M. A Systematic Investigation of Exotic Matter in Neutron Stars. Ph.D. Thesis, Claremont Graduate University, Claremont, CA, USA, San Diego State University, San Diego, CA, USA, 2017.

8. Kaminker, A.D.; Pethick, C.J.; Potekhin, A.Y.; Yakovlev, D.G. Neutrino-pair bremsstrahlung by electrons in neutron star crusts. Astron. Astrophys. 1999, 343, 1009-1024.

9. Flowers, E. Neutrino-pair emission in dense matter: A many-body approach. Astrophys. J. 1973, 180, 911-936. [CrossRef]

10. Itoh, N.; Kohyama, Y. Neutrino-pair bremsstrahlung in dense stars. I-Liquid metal case. Astrophys. J. $1983,275,858-866$.

11. Itoh, N.; Matsumoto, N.; Seki, M.; Kohyama, Y. Neutrino-pair bremsstrahlung in dense stars. II—Crystalline lattice case. Astrophys. J. 1984, 279, 413-418. [CrossRef]

12. Itoh, N.; Kohyama, Y.; Matsumoto, N.; Seki, M. Neutrino-pair bremsstrahlung in dense stars. III-Low-temperature quantum corrections in the liquid metal phase. Astrophys. J. 1984, 280, 787-791. [CrossRef]

13. Itoh, N.; Kohyama, Y.; Matsumoto, N.; Seki, M. Neutrino-pair bremsstrahlung in dense stars. IV—Phonon contributions in the crystalline lattice phase. Astrophys. J. 1984, 285, 304-311. [CrossRef]

14. Pethick, C.J.; Thorsson, V. Effects of electron band structure on neutrino pair bremsstrahlung in neutron star crusts. Phys. Rev. D 1997, 56, 7548-7558. [CrossRef] 
15. Na, X.; Xu, R.; Weber, F.; Negreiros, R. On the Transport Properties of a Quark-Hadron Coulomb Lattice in the Cores of Neutron Stars. Phys. Rev. C 2012, 86, 123016. [CrossRef]

16. Antoniadis, J.; Freire, P.C.C.; Wex, N.; Tauris, T.M.; Lynch, R.S.; Kerkwijk, M.H.; Kramer, M.; Bassa, C.; Dhillon, V.S.; Driebe, T;; et al. A Massive Pulsar in a Compact Relativistic Binary. Science 2013, 340, 1233232. [CrossRef] [PubMed]

17. Scarpettini, A.; Gómez Dumm, D.; Scoccola, N.N. Light pseudoscalar mesons in a nonlocal SU(3) chiral quark model. Phys. Rev. C 2004, 69, 114018.

18. Contrera, G.A.; Gómez Dumm, D.; Scoccola, N.N. Nonlocal SU(3) chiral quark models at finite temperature: The role of the Polyakov loop. Phys. Lett. B 2008, 661, 113-117. [CrossRef]

19. Contrera, G.A.; Gómez Dumm, D.; Scoccola, N.N. Meson properties at finite temperature in a three flavor nonlocal chiral quark model with Polyakov loop. Phys. Rev. C 2010, 81, 054005. [CrossRef]

20. Orsaria, M.; Rodrigues, H.; Weber, F.; Contrera, G.A. Quark-hybrid matter in the cores of massive neutron stars. Phys. Rev. D 2013, 87, 023001. [CrossRef]

21. Orsaria, M.; Rodrigues, H.; Weber, F.; Contrera, G.A. Quark deconfinement in high-mass neutron stars. Phys. Rev. C 2014, 89, 015806. [CrossRef]

22. Ranea-Sandoval, I.F.; Han, S.; Orsaria, M.G.; Contrera, G.A.; Weber, F.; Alford, M.G. Constant-sound-speed parametrization for Nambu-Jona-Lasinio models of quark matter in hybrid stars. Phys. Rev. C 2016, 93, 045812. [CrossRef]

23. Boguta, J.; Bodmer, A.R. Relativistic calculation of nuclear matter and the nuclear surface. Nucl. Phys. A 1977, 292, 413-428. [CrossRef]

24. Boguta, J.; Stöcker, H. Systematics of nuclear matter properties in a non-linear relativistic field theory. Phys. Lett. B 1983, 120, 289-293. [CrossRef]

25. Mellinger, R.D., Jr.; Weber, F.; Spinella, W.; Contrera, G.A.; Orsaria, M.G. Quark Deconfinement in Rotating Neutron Stars. Universe 2017, 3, 5. [CrossRef]

26. Typel, S.; Ropke, G.; Klahn, T.; Blaschke, D.; Wolter, H.H. Composition and thermodynamics of nuclear matter with light clusters. Phys. Rev. C 2010, 81, 015803. [CrossRef]

27. Lalazissis, G.A.; Niksic, T.; Vretenar, D.; Ring, P. New relativistic mean-field interaction with densitydependent meson-nucleon couplings. Phys. Rev. C 2005, 71, 024312. [CrossRef]

28. Fuchs, C.; Lenske, H.; Wolter, H.H. Density dependent hadron field theory. Phys. Rev. C 1995, $52,3043$. [CrossRef]

29. Typel, S.; Wolter, H.H. Relativistic mean field calculations with density-dependent meson-nucleon coupling. Nucl. Phys. A 1999, 656, 331-364. [CrossRef]

30. Typel, S. Relativistic model for nuclear matter and atomic nuclei with momentum-dependent self-energies. Phys. Rev. C 2005, 71, 064301. [CrossRef]

31. Rijken, T.A.; Nagels, M.M.; Yamamoto, Y. Baryon-Baryon Interactions: Nijmegen Extended-Soft-Core Models. Prog. Theor. Phys. Suppl. 2010, 185, 14-71. [CrossRef]

32. Miyatsu, T.; Cheoun, M.; Saito, K. Equation of state for neutron stars in SU(3) flavor symmetry. Phys. Rev. C 2013, 88, 015802. [CrossRef]

33. Yasutake, N.; Lastowiecki, R.; Benic, S.; Blaschke, D.; Maruyama, T.; Tatsumi, T. Finite-size effects at the hadron-quark transition and heavy hybrid stars. Phys. Rev. C 2014, 89, 065803. [CrossRef]

34. Ogata, S.; Ichimaru, S. Critical examination of $\mathrm{N}$ dependence in the Monte Carlo calculations for a classical one-component plasma. Phys. Rev. A 1987, 36, 5451. [CrossRef]

35. Haensel, P.; Potekhin, A.Y.; Yakovlev, D.G. Neutron Stars 1: Equation of State and Structure; Springer: New York, NY, USA, 2007.

36. Baiko, D.A.; Yakovlev, D.G. Thermal and electrical conductivities of Coulomb crystals in neutron stars and white dwarfs. Astron. Lett. 1995, 21, 702-709.

37. Haensel, P.; Kaminker, A.D.; Yakovlev, D.G. Electron neutrino-antineutrino bremsstrahlung in a liquid phase of neutron star crusts. Astron. Astrophys. 1996, 314, 328-340.

38. Baiko, D.A.; Kaminker, A.D.; Potekhin, A.Y.; Yakovlev, D.G. Ion Structure Factors and Electron Transport in Dense Coulomb Plasmas. Phys. Rev. Lett. 1998, 81, 5556-5559. [CrossRef]

39. Raikh, M.E.; Yakovlev, D.G. Thermal and electrical conductivities of crystals in neutron stars and degenerate dwarfs. Astrophys. Space Sci. 1982, 87, 193-203. [CrossRef]

40. Ziman, J.M. Principles of the Theory of Solids; Cambridge University Press: Cambridge, UK, 1972. 
41. Mochkovitch, R.; Hansen, J.P. Fluid-solid coexistence curve of dense coulombic matter. Phys. Lett. 1979, 73A, 35-38. [CrossRef]

42. Lamb, D.Q.; Lattimer, J.M.; Pethick, C.J.; Ravenhall, D.G. Physical properties of hot, dense matter: The bulk equilibrium approximation. Nucl. Phys. A 1981, 360, 459-482. [CrossRef]

43. Ravenhall, D.G.; Pethick, C.J.; Wilson, J.R. Structure of Matter below Nuclear Saturation Density. Phys. Rev. Lett. 1983, 50, 2066-2069. [CrossRef]

44. Williams, R.D.; Koonin, S.E. Sub-saturation phases of nuclear matter. Nucl. Phys. A 1985, 435, $844-858$. [CrossRef]

45. Lorenz, C.P.; Ravenhall, D.G.; Pethick, C.J. Neutron star crusts. Phys. Rev. Lett. 1993, 70, 379-382. [CrossRef] [PubMed]

46. Pethick, C.J.; Thorsson, V. Neutrino pair bremsstrahlung in neutron star crusts: A reappraisal. Phys. Rev. Lett. 1994, 72, 1964-1967. [CrossRef] [PubMed]

(C) 2018 by the authors. Licensee MDPI, Basel, Switzerland. This article is an open access article distributed under the terms and conditions of the Creative Commons Attribution (CC BY) license (http:/ / creativecommons.org/licenses/by/4.0/). 